\title{
PRKAG2 is differentially expressed in brain metastatic human breast cancer.
}

Shahan Mamoor, MS ${ }^{1}$

$3 \quad$ 'shahanmamoor@gmail.com East Islip, NY USA

Metastasis to the brain is a clinical problem in patients with breast cancer ${ }^{1-3}$. We mined published microarray data ${ }^{4,5}$ to compare primary and metastatic tumor transcriptomes for the discovery of genes associated with brain metastasis in humans with metastatic breast cancer. We found that 5'-AMP-activated protein kinase subunit gamma-2, encoded by PRKAG2, was among the genes whose expression was most different in the brain metastases of patients with metastatic breast cancer as compared to primary tumors of the breast. PRKAG2 mRNA was present at decreased quantities in brain metastatic tissues as compared to primary tumors of the breast. Importantly, expression of PRKAG2 in primary tumors was significantly correlated with patient overall survival. Modulation of PRKAG2 expression may be relevant to the biology by which tumor cells metastasize from the breast to the brain in humans with metastatic breast cancer.

Keywords: breast cancer, metastasis, brain metastases, central nervous system metastases, therapeutics in breast cancer. 
One report described a 34\% incidence of central nervous system metastases in patients treated with trastuzumab for breast cancer ${ }^{2}$. More recently, the NEfERT-T clinical trial ${ }^{6}$ which compared administration of either neratinib or trastuzumab in conjunction with paclitaxel demonstrated that in a randomized, controlled setting, in breast cancer patients treated with neratinib, not only was the incidence of central nervous system recurrence significantly lower, the time to central nervous system metastasis was significantly delayed as compared to patients administered trastuzumab ${ }^{6}$. The alarmingly high rate of central nervous system metastasis described, as well as data, both anecdotal ${ }^{2}$ and from a randomized, controlled setting ${ }^{6}$ illustrating that treatment with trastuzumab may be associated with these events demands an enhanced understanding of the transcriptional makeup of brain metastatic tissues to support identification of therapeutic targets, whether they are treatment related or not. We performed a global comparative analysis of primary and metastatic tumors in patients with brain metastatic breast cancer ${ }^{4,5}$. We discovered differential and decreased expression of the gene encoding 5'-AMP-activated protein kinase subunit gamma-2, PRKAG2, in brain metastatic tissues of patients with metastatic breast cancer.

\section{$\underline{\text { Methods }}$}

We used datasets GSE10893 ${ }^{4}$ and GSE52604 5 for this global differential gene expression analysis of brain metastatic breast cancer in conjunction with GEO2R. GSE10893 was generated using Agilent-011521 Human 1A Microarray G4110A technology with $n=11$ primary breast tumors and $n=4$ brain metastases from patients with breast cancer; analysis was performed using platform GPL885. GSE52604 was generated using Agilent-014850 Whole Human Genome Microarray 4x44K G4112F with $n=10$ normal breast tissues and $n=35$ brain metastases from patients with breast cancer; analysis was performed using platform GPL6480. The Benjamini and Hochberg method of $p$-value adjustment was used for ranking of differential expression but raw $p$-values were used to assess statistical significance of global differential expression. Log-transformation of data was auto-detected, and the NCBI generated category of platform annotation was used. A statistical test was performed to evaluate whether PRKAG2 gene expression was significantly different between primary tumors of the breast and brain metastases in humans with breast cancer using a two-tailed t-test. For Kaplan-Meier survival analysis, we used the Kaplan-Meier plotter online tool ${ }^{7}$ for correlation of PRKAG2 mRNA expression levels with overall survival in $n=1402$ breast cancer patients.

\section{$\underline{\text { Results }}$}

We performed global comparative transcriptome analysis of metastatic tumor tissues of patients with metastatic breast cancer using published microarray data ${ }^{4,5}$ to describe the transcriptional landscape of brain metastasis in human breast cancer in an unbiased fashion and for the discovery of novel therapeutic targets.

\section{PRKAG2 is differentially expressed in the brain metastases of patients with brain metastatic breast} cancer.

Through blind, systems-level analysis of published microarray data ${ }^{4}$, we identified the 5'-AMP-activated protein kinase subunit gamma-2, encoded by PRKAG2, as a differentially expressed gene in the breast metastatic tissues of humans with breast cancer (Table 1). When sorting each of the genes expressed in brain metastases based on significance of difference as compared to primary tumors of the breast in patients with breast cancer, PRKAG2 ranked 138 out of 17418 total transcripts (Chart 1), equating to $99.2 \%$ differential expression. Differential expression of PRKAG2 in the brain metastases of patients with metastatic breast cancer was statistically significant (Chart $1 ; p=3.12 \mathrm{e}-04$ ). 
Differential gene expression analyses that utilize primary and metastatic tumor tissues to discover genes associated with metastasis can be challenging due to the relatively heterogenous cellular composition and constitution of the tumor as compared to normal, untransformed (benign) tissues. Thus, to attempt to validate differential transcriptome-wide differential expression of PRKAG2 in brain metastases in human metastatic breast cancer, we queried a second microarray dataset ${ }^{5}$, here comparing normal breast tissues rather than primary tumors of the breast to brain metastases. Again, we identified PRKAG2 as a differentially expressed gene in the brain metastatic tissues of patients with breast cancer (Chart 2). When sorting each of the genes expressed in brain metastases based on significance of difference as compared to normal breast tissues, PRKAG2 ranked 17870 out of 41093 total transcripts (Chart 2), equating to $56.5 \%$ differential expression. Differential expression of PRKAG2 in the brain metastases of patients with metastatic breast cancer approached statistical significance (Chart 2; $p=5.64 \mathrm{e}-02$ ). Thus, differential expression of PRKAG2, transcriptome-wide, in the brain metastases of women with metastatic breast cancer was conserved across two independent microarray datasets, both when compared to benign and transformed breast tissues. This suggests that PRKAG2 differential expression in human brain metastatic breast cancer is a bona fide biological process.

\section{PRKAG2 is expressed at lower levels in the brain metastases of patients with metastatic breast} cancer.

We obtained exact mRNA expression levels for PRKAG2, in primary tumors of the breast and in brain metastasis of patients with brain metastatic breast cancer to determine direction and statistical significance of change in PRKAG2 expression in brain metastatic tissues. We observed significantly lower expression of PRKAG2 in the brain metastases of patients with breast cancer as compared to primary tumors of the breast: PRKAG2 was expressed at $0.89 \pm 0.51$ arbitrary units (AU) in primary tumors of the breast, while it was expressed at $-0.46 \pm 0.54 \mathrm{AU}$ in brain metastatic tissues. The difference in PRKAG2 mRNA levels between primary tumors of the breast and brain metastatic tissues was statistically significant (Figure 1; $p=0.000662$ ).

\section{PRKAG2 expression is significantly correlated with survival outcomes in human breast cancer.}

We performed Kaplan-Meier survival analysis ${ }^{7}$ in $n=1402$ breast cancer patients in total, to evaluate whether PRKAG2 tumor expression was correlated with survival outcomes in breast cancer. We observed a statistically significant correlation between primary tumor expression of PRKAG2 and overall survival (OS) in patients with breast cancer, in the upper survival quartile (Figure 2). Patients whose primary tumors expressed low levels of PRKAG2 possessed median OS of 90 months, while patients whose tumors expressed high levels of PRKAG2 possessed median OS of 143 months. This difference in OS based on PRKAG2 tumor expression in patients with breast cancer was statistically significant (Figure 2, Chart 3; logrank $p$-value 0.037; hazard ratio: 0.75 (0.57-0.98)).

Thus, by mining published microarray data ${ }^{4,5}$ in an unbiased and systematic fashion, we identified 5'-AMP-activated protein kinase subunit gamma-2, encoded by PRKAG2, as among the genes whose expression was most different, transcriptome-wide, in the brain metastases of patients with breast cancer when compared to primary tumors of the breast; we observed significantly decreased expression of PRKAG2 in brain metastases as compared to primary tumors of the breast. Further, we found a significant correlation between PRKAG2 expression and patient survival outcomes, as overall survival was significantly greater in patients whose primary tumors expressed higher levels of PRKAG2 as compared to patients whose primary tumors expressed lower levels of PRKAG2. 


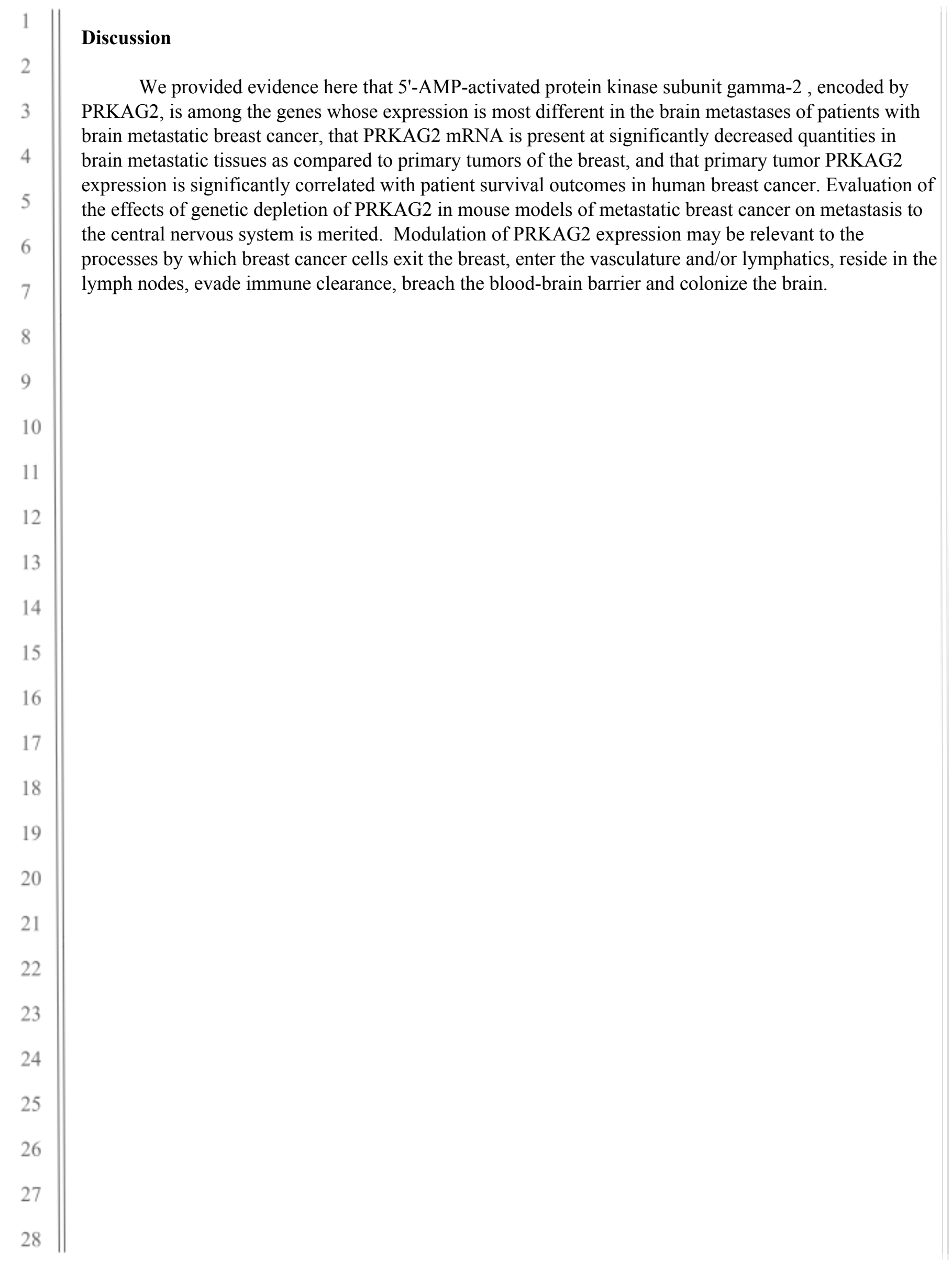




\section{References}

1. Lin, N.U., Amiri-Kordestani, L., Palmieri, D., Liewehr, D.J. and Steeg, P.S., 2013. CNS metastases in breast cancer: old challenge, new frontiers.

2. Bendell, J.C., Domchek, S.M., Burstein, H.J., Harris, L., Younger, J., Kuter, I., Bunnell, C., Rue, M., Gelman, R. and Winer, E., 2003. Central nervous system metastases in women who receive trastuzumab-based therapy for metastatic breast carcinoma. Cancer, 97(12), pp.2972-2977.

3. Tsukada, Y., Fouad, A., Pickren, J.W. and Lane, W.W., 1983. Central nervous system metastasis from breast carcinoma autopsy study. Cancer, 52(12), pp.2349-2354.

4. Weigman, V.J., Chao, H.H., Shabalin, A.A., He, X., Parker, J.S., Nordgard, S.H., Grushko, T., Huo, D., Nwachukwu, C., Nobel, A. and Kristensen, V.N., 2012. Basal-like Breast cancer DNA copy number cancer research and treatment, 133(3), pp.865-880.

5. Salhia, B., Kiefer, J., Ross, J.T., Metapally, R., Martinez, R.A., Johnson, K.N., DiPerna, D.M., Paquette, K.M., Jung, S., Nasser, S. and Wallstrom, G., 2014. Integrated genomic and epigenomic analysis of breast cancer brain metastasis. Plos one, 9(1), p.e85448.

6. Awada, A., Colomer, R., Inoue, K., Bondarenko, I., Badwe, R.A., Demetriou, G., Lee, S.C., Mehta, A.O., Kim, S.B., Bachelot, T. and Goswami, C., 2016. Neratinib plus paclitaxel vs trastuzumab plus paclitaxel in previously untreated metastatic ERBB2-positive breast cancer: the NEfERT-T randomized clinical trial. JAMA oncology, 2(12), pp.1557-1564.

7. Györffy, B., Lanczky, A., Eklund, A.C., Denkert, C., Budczies, J., Li, Q. and Szallasi, Z., 2010. An online survival analysis tool to rapidly assess the effect of 22,277 genes on breast cancer prognosis using microarray data of 1,809 patients. Breast cancer research and treatment, 123(3), pp.725-731. 


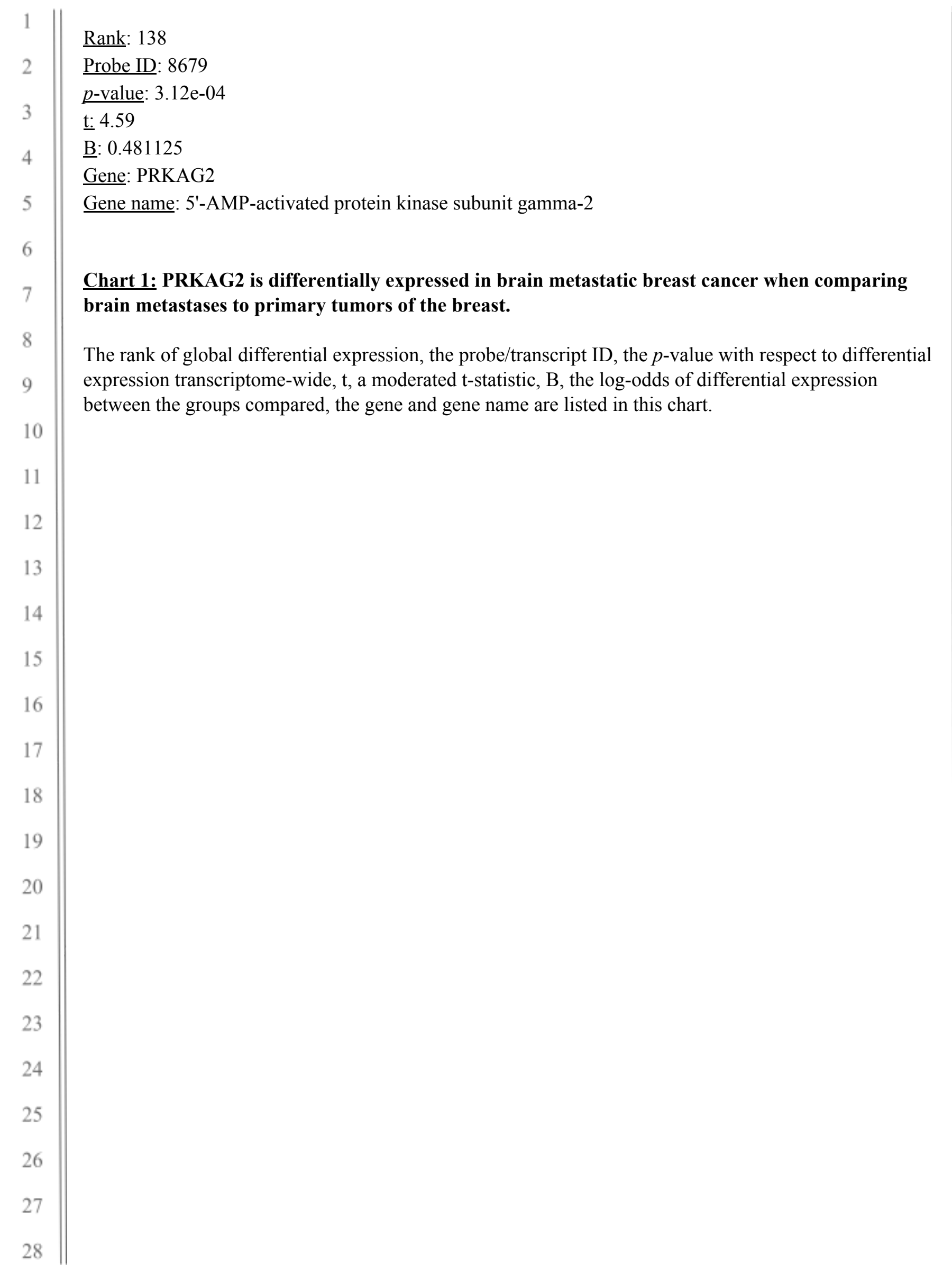




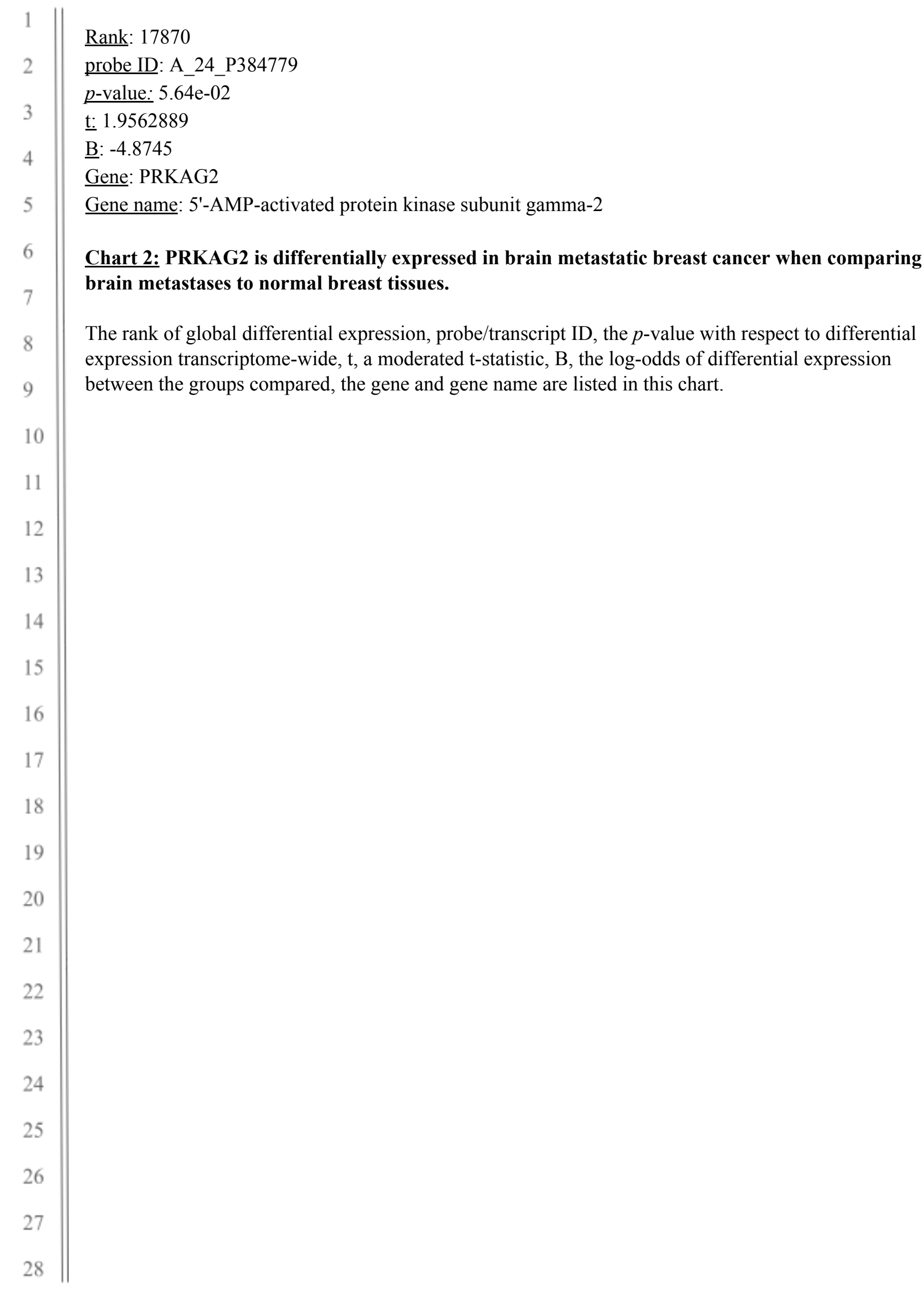




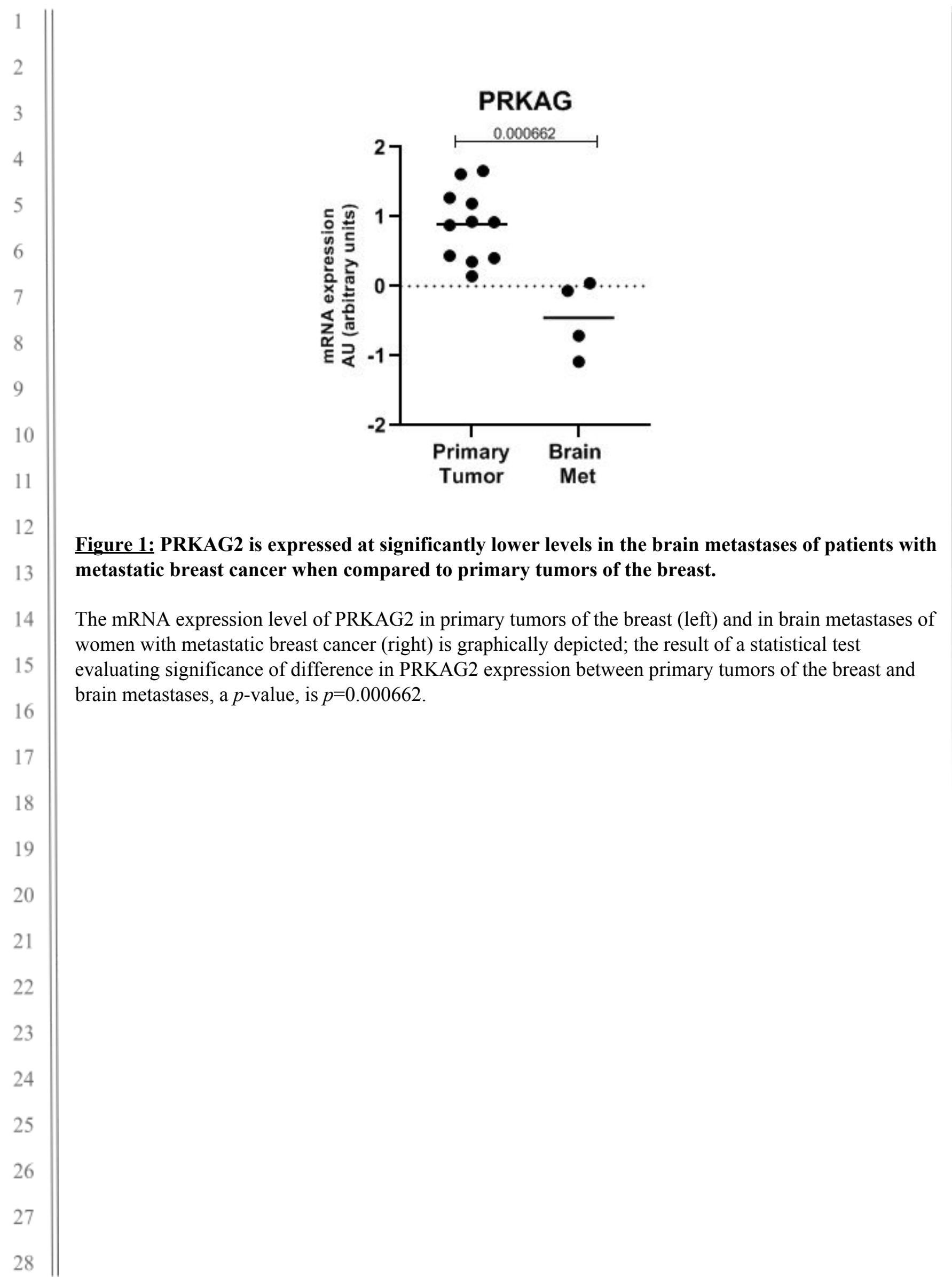




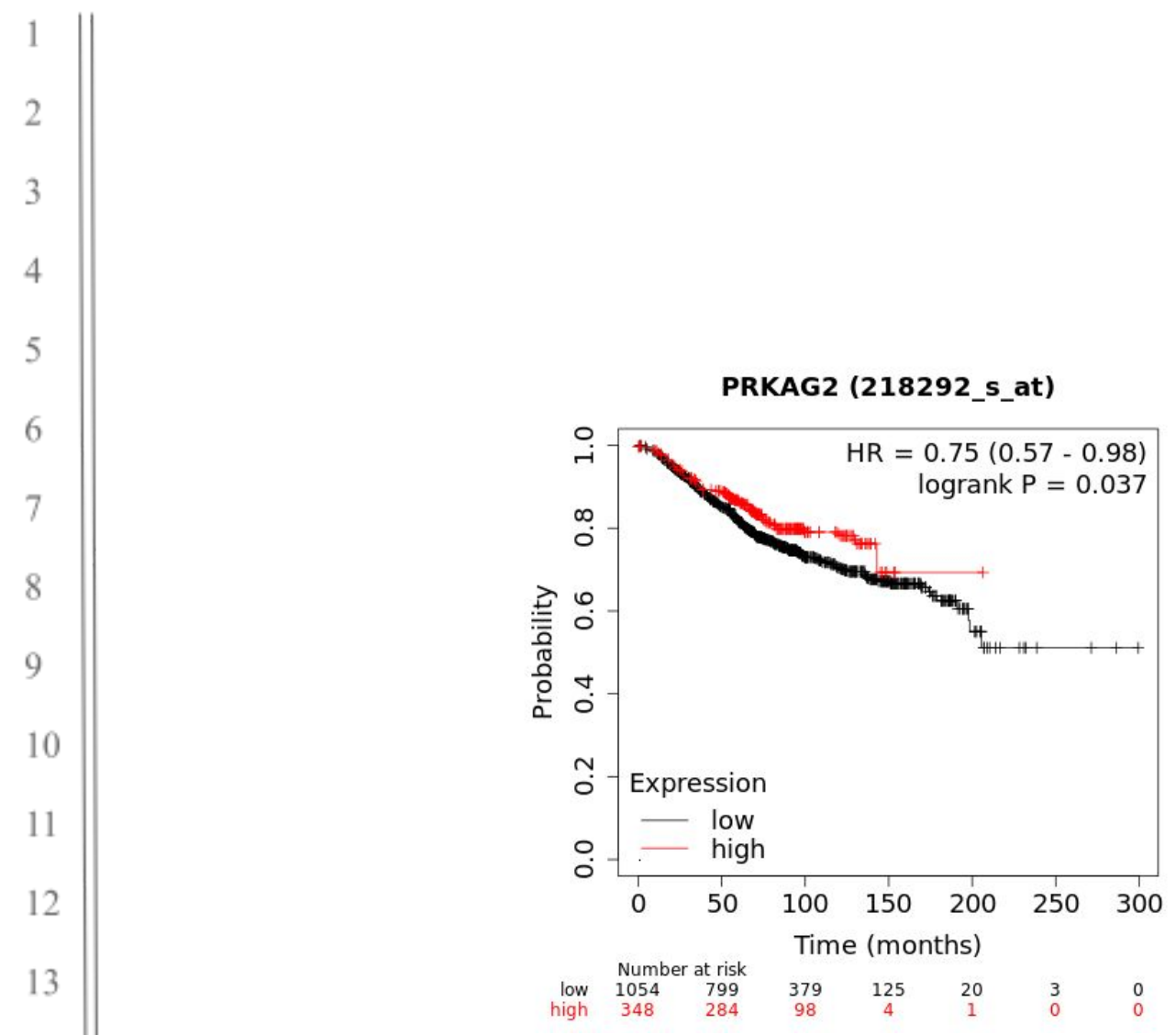

Figure 2: Significant correlation between PRKAG2 primary tumor expression and overall survival in patients with breast cancer.

Depicted in this Kaplan-Meier plot is the probability of overall survival for $n=1402$ total patients stratified into two groups, based on low or high expression of PRKAG2 in patient primary tumors, in the upper survival quartile. The log rank $p$-value denoting statistical significance of difference in overall survival when comparing the two groups, as well as hazard ratio for this comparison is listed above. Listed below is the number of patients at risk (number of patients alive) per interval, after stratification based on PRKAG2 expression; in the first interval, number at risk is number of patients alive; in each subsequent interval, number at risk is the number at risk less those who have expired or are censored. 


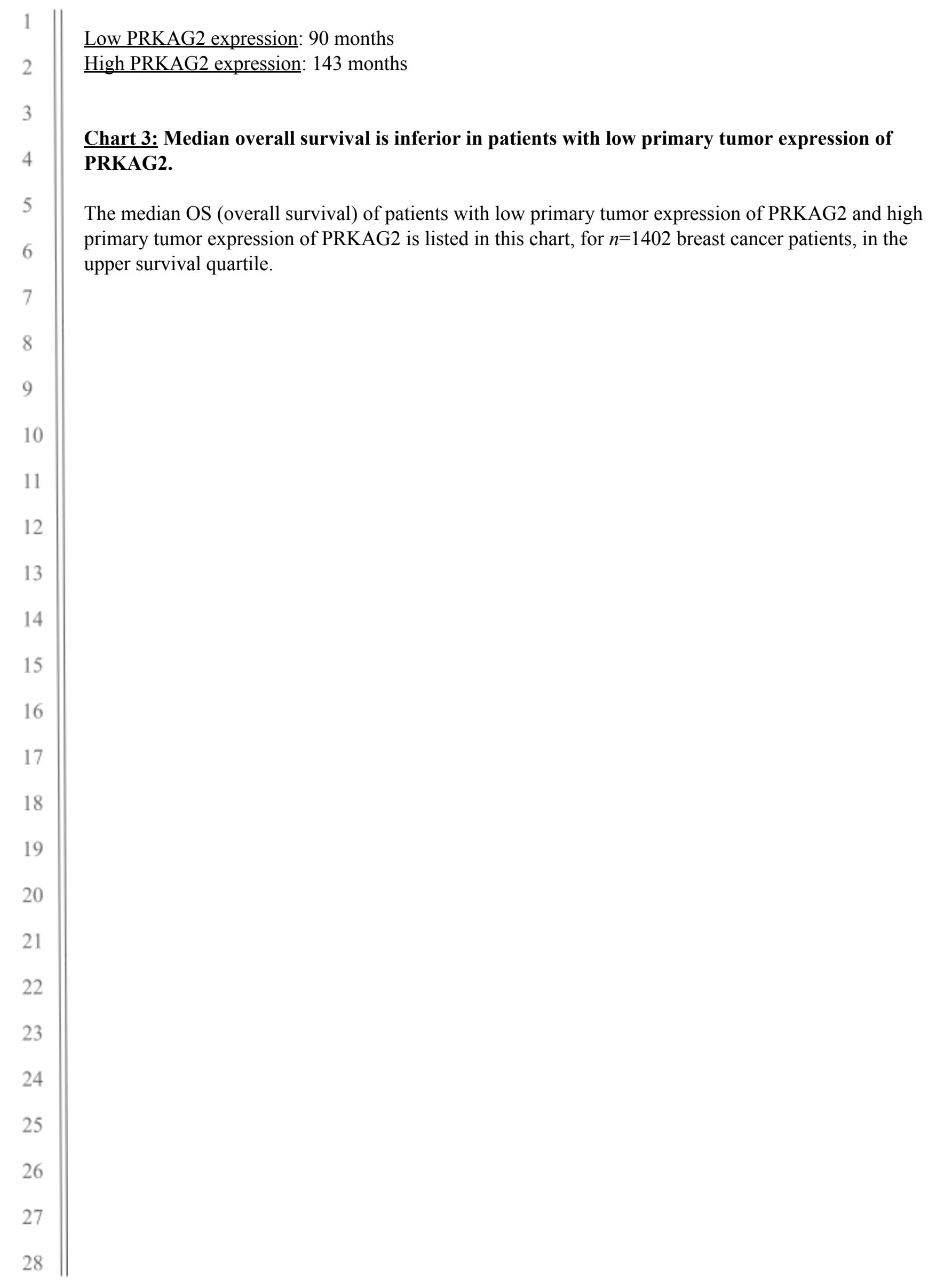

